case report

\title{
Adenocarcinoma of the small bowel
}

\author{
Metka Šavli, Breda Jamar \\ Institute of Clinical Radiology, University Medical Centre, Ljubljana, Slovenia
}

Background. Adenocarcinoma of small bowel is generally a rather rare primary tumour of small bowel with a prevalence rate of 0.5 - 3.0 / 100.000 population, but the most frequent tumour of small intestine. It more often involves the duodenum and jejunum than the ileum. The aim of this paper is also to point out the value of small bowel follow through (SBFT) in the diagnosis of stenosing lesions.

Case report. An 83 - year old male patient suffered from abdominal pain, malaise, vomiting, cachexia and diarrhoea for 3 months. The result of occult blood testing was negative. Haemoglobin level was normal. Proctoscopy, colonoscopy, upper gastrointestinal (GI) endoscopy, and ultrasonography (US) did not explain the patient's problems. Ileus of the small bowel was established with abdominal plain film. Small bowel follow through (SBFT) and computer tomography (CT) showed a stenosing tumour in the jejunum. Adenocarcinoma of the small bowel was established with histological examination after resection of the tumor.

Conclusions. SBFT, with manual compression of all segments of the small bowel, can be a very accurate diagnostic investigation for evaluation of stenosing lesions in this part of the intestine.

Key words: intestinal obstruction; jejunal neoplasms; adenocarcinoma - radiography

\section{Introduction}

Comprising about $2 \%$ of all gastrointestinal malignancies, malignant tumors of the small bowel are relatively rare ${ }^{1}$, adenocarcinomas being the most frequent among them. Their peak incidence, slightly higher in males than in females, is in the 7 th decade. ${ }^{2}$ More often they are found in the jejunum or duodenum. In the jejunum, adenocarcinomas are usually located within

Received 14 May 2007

Accepted 5 June 2007

Correspondence to: Breda Jamar MD, Institute of Clinical Radiology, University Medical Centre, Zaloška 7, SI-1000 Ljubljana, Slovenia. Tel. +386 1522 8530; Fax: +386 1522 2497; E-mail: breda.jamar@kclj.si the first $30 \mathrm{~cm}$ distally of the ligament of Treitz. ${ }^{3}$ Lymphatic spread to the regional lymph nodes and through the portal system to the liver are frequent. Peritoneal metastases can also be found, or there may be direct progression of the tumor into the adjoining structures. ${ }^{4}$ Adult coeliac disease, Crohn's disease and Peutz - Jeghers syndrome are precancerous conditions. ${ }^{5-7}$

More specific symptoms are preceded by a period of vague abdominal discomfort, dyspepsia and malaise, often not alarming enough for the patient to seek medical advice. The more specific clinical presentation is associated with obstruction and ulceration of the cancer. Since the small bowel contents are liquid, obstructive symptoms 
are somewhat late and may diminish to conservative treatment.

Because of its anatomy, small bowel is difficult to examine. The proximal jejunum and terminal ileum can be examined with enteroscopy, while for the stenosing processes in the mesenteric small intestine, radiologic examinations, especially dedicated SBFT and CT, are dominant diagnostic procedures. ${ }^{8,9}$ On SBFT, the typical image of primary adenocarcinoma of the small intestine is an annular lesion of the " apple core » type. ${ }^{2}$ It is usually symmetric, with a centrally positioned stricture. It is rigid and its shape is not significantly changed during compression of the abdomen. ${ }^{10}$

\section{Case report}

An 83-year-old man with arterial hypertension was first referred to the proctologist because of difficult defecation, abdominal pain and diarrhoea lasting for about one month. On proctoscopy, polyp of 5 - times $5 \mathrm{~mm}$ was found in the rectum. It was resected at a later colonoscopy and sent for histological examination. Some diverticula were also observed. With the upper GI endoscopy signs of chronic atrophic gastritis were found. With abdominal ultrasonography small cysts in the right kidney were revealed. However, the results of all examinations could not explain the patient's problems. Two months later the patient returned to the emergency department, for the third time in one month, because of severe vomiting and weight loss. Haemoglobin level was found normal. Dilated small bowel was observed on abdominal plain film (Figure 1), while no signs of acute abdomen were seen on physical examination, leading abdominal surgeons at the first two patient's visits to conclusion that there was no indication for surgery. At the third patient's visit to the

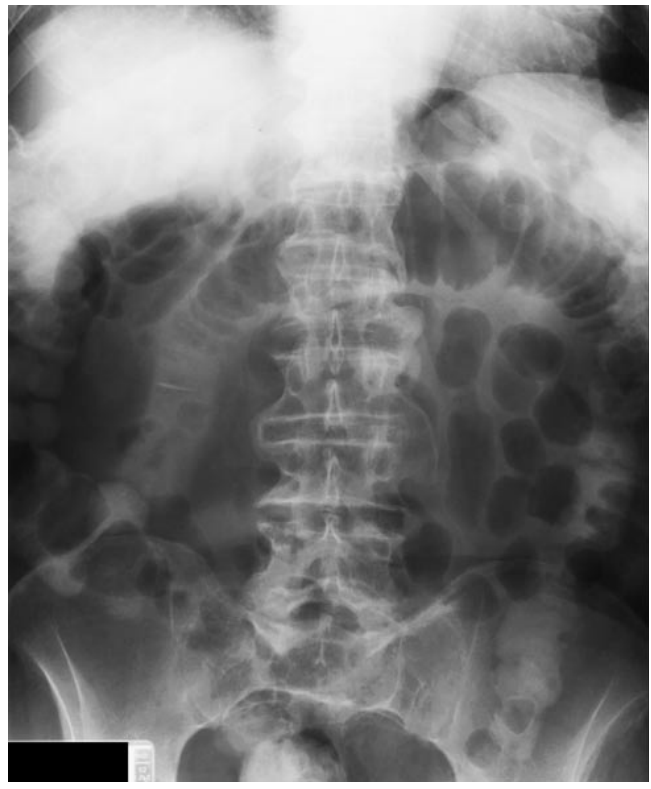

Figure 1. Abdominal plain film: dilated small bowel loops - obstruction.

emergency department, the gastroenterologist referred him to SBFT.

On SBFT, the jejunum was found dilated, and an oval formation, approximately 3 - times $3 \mathrm{~cm}$ in size in the distal jejunum, causing relative obstruction, was revealed. Proximally to the formation, in a length of about $30 \mathrm{~cm}$, thickened mucosal folds and irregular bowel wall were seen (Figure 2 ). The shape of the segment did not significantly change during compression of the abdomen. The process was obviously malignant, not inflammatory. As definite diagnosis could not be established, CT scan of the abdomen was done. It showed a solid tumour in the jejunum, 3 - times $3 \mathrm{~cm}$ in size (Figure 3,4). There were no signs of spread into adjacent mesenteric fat. Radiologic signs of mechanical obstruction (dilated small bowel) could still be seen. No lymphadenopathy was found. With the exception of right cystic kidney, parenchymal organs were normal. There was some free fluid in the Proust's pouch. 


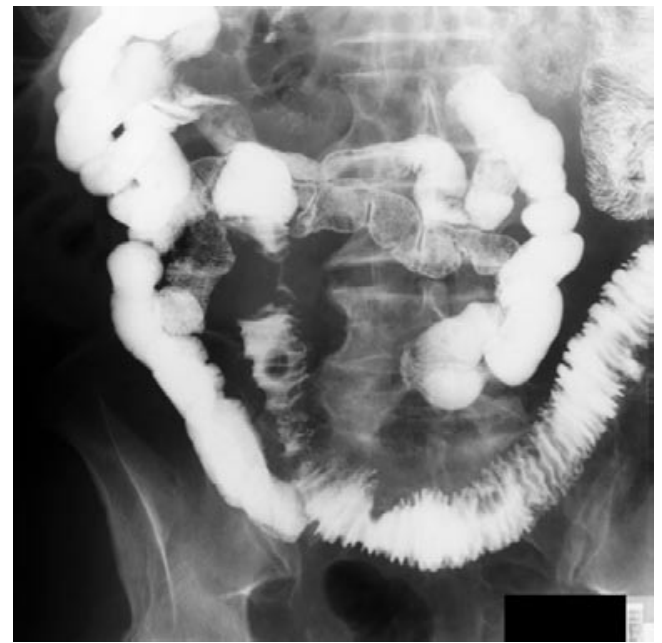

Figure 2. SBFT: Stenosis and an oval formation in the distal jejunum, causing a relative obstruction, thickened mucosal folds and irregular bowel wall proximally .

The patient was admitted to surgery. Implantation of pace maker for his arrhythmia was needed before he could be operated upon (5 days later). A $30 \mathrm{~cm}$ long segment of the jejunum was resected. Appendectomy was also performed. The histopathologic diagnosis was adenocarcinoma of the small bowel. One out of 17 nodes in the mesentery was found malignant. Lymphangiocarcinomatosis of the mesentery was also established.

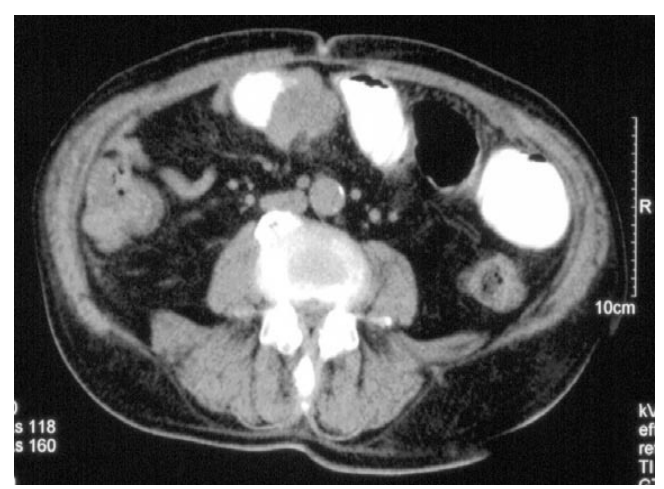

\section{Discussion}

The performed examinations of the proximal and distal part of the alimentary tract - proctoscopy, colonoscopy, upper GI endoscopy - could not explain the patient's problems. Abdominal US revealed small cysts in the right kidney. The small bowel and colon attracted no attention of the ultrasonographist. It should be born in mind, however, that the interpretation of the bowel US is highly operator - dependent. Vast experience is needed to achieve accuracy rates comparable with those from the literature. ${ }^{6}$

On the three patient's visits of the emergency department in just one month because of the clinical progression of his state (severe vomiting, abdominal pain and loss of weight), signs of the small bowel mechanical ileus could be demonstrated with plain film of the abdomen, while no signs of acute abdomen could be seen on physical examination. The levels of haemoglobin were normal.

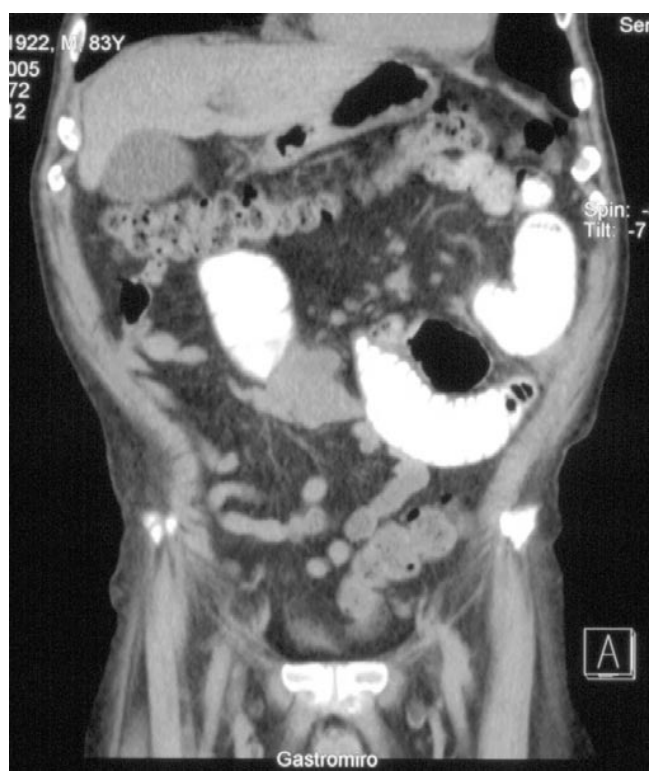

Figure 3 and 4. Computed tomography: A solid tumour in the jejunum. Dilated small bowel loops proximally. 
In order to examine the mesenteric small bowel, a dedicated investigation, the SBFT, was indicated. The revealed oval formation, approximately 3 - times $3 \mathrm{~cm}$ in size, in the distal part of the jejunum, causing relative obstruction and thickened mucosal folds as well as irregular bowel wall proximally to the obstruction not changing during abdominal compression, could be recognised as malignant. As the changes were not typical of the small bowel adenocarcinoma, CT scan of the abdomen was performed for the differential diagnosis of lymphoma.

CT disclosed a solid tumour in the jejunum causing a relative obstruction and, proximally, dilatation of a small bowel loop. There was some free fluid in the pouch of Proust, but no signs of distal spread. The question whether it was a primary tumour or a direct progression from surroundings could not be answered.

Because of the radiologic signs of obstructive ileus, the results of SBFT and CT, the patent was admitted for surgery. A 30 $\mathrm{cm}$ long segment of the jejunum was resected. Appendectomy was also performed. The final diagnosis of adenocarcinoma of the small bowel was established with histopathologic examination.

\section{Conclusion}

Abdominal pain, vomiting, anaemia, and the presence of dilated proximal jejunum should suggest an obstructing neoplasm of the small bowel in older patients, and indicate the need for further diagnostic procedure with fluoroscopic small bowel study. The demonstration of a small bowel stenosing lesion depends primarily on SBFT. In most cases, the differentiation between malign or benign (e.g. inflammatory) lesion can be made, although the final diagnosis of the type of malignancy is done by histopathology.

\section{References}

1. Barclay THC, Shapira DV. Malignant tumors of the small intestine. Cancer 1983; 51: 878-81.

2. Dean DT, Maglinte MD. Malignant tumors of the small bowel: In Gore RM, Levine MS, editors. Textbook of gastrointestinal radiology. Philadelphia: WB Saunders Company; 2000. p. 792-9.

3. Herbsman H, Wetstein L. Tumors of the small intestine. Curr Probl Surg 1980; 17: 121-82.

4. Lightdale CJ, Sherlock P. Small intestinal tumors (other than lymphoma and carcinoid). In Berk JE, editor. Bockus Gastroenterology. Philadelphia: WB Saunders Company 1985; p.1887-99.

5. Berstein D, Rogers A. Malignancy in Crohn's disease. Am J Gastroenterol 1996; 91: 434-40.

6. Lashner BA. Risk factors for small bowel cancer in Crohn's disease. Dig Dis Sci 1992; 37: 1179-84.

7. Spigelman AD, Murday V, Phillips RKS. Cancer and the Peutz - Jeghers syndrome. Gut 1989; 30: 1588-90.

8. Bessette JR, Maglint DDT, Kelvin FM, et al. Primary malignant tumors in the small bowel: a comparison of the small bowel enema and conventional follow - through examination. Am J Roentgenol 1989; 153: 741-4.

9. Hulnick DH, Megibow AJ. Computed tomography of the small bowel. In: Herlinger H, Maglinte D, eds. Clinical Radiology of the Small Intestine. Philadelphia: WB Saunders; 1989: p.161-200.

10. Papadopoulos VD, Nolan DJ. Carcinoma of the small intestine. Clin Radiol 1985; 36: 409-13. 